\title{
TOWARDS WAVE DISTURBANCE IN PORTS COMPUTED BY A DETERMINISTIC CONVOLUTION-TYPE MODEL
}

\begin{abstract}
Hemming A. Schäffer ${ }^{1}$
Among the wide range of potential applications of the convolution-type approach to deterministic wave modeling, this paper looks into the challenge of complex shaped domains. The canonical case of diffraction around a semiinfinite vertical barrier, the 'Sommerfeld diffraction' case, is first studied. Focusing on locally constant water depth, the convolution method is related to a boundary integral representation by which the impulse response function representing the convolution kernel is related to a Green's function for the Laplace equation. This provides a framework for determining the impulse response function by solving a local, three-dimensional Laplace problem prior to the time-stepping of the wave transformation problem. For the Sommerfeld case, numerical results for the impulse response function near the barrier are computed numerically and compared with an analytical solution. For complex-shaped domains, numerical determination of the impulse response functions is the only solution. A very preliminary example of application to wave disturbance in a real port is given.
\end{abstract}

Keywords: convolution-type model, wave agitation, diffraction, Sommerfeld, boundary integral equation

\section{INTRODUCTION TO THE CONVOLUTION-TYPE APPROACH}

\section{Scope}

The convolution-type approach to deterministic wave modeling has the potential to result in preferred models for port, coastal, and offshore applications. The challenges are many, but the prospective benefits are significant. This paper presents a step forward towards the efficient modeling of nonlinear, fully dispersive, irregular, multidirectional waves as they transform over several kilometers of variable bathymetry and interact with fixed or floating structures in complex-shaped domains. Various developments in this direction can be found in papers by the author at previous Coastal Engineering Conferences and elsewhere, see e.g. http://www.SchafferWaves.dk/publications/

\section{Approach}

Like many other wave models, the present approach rests on the time-integration of the dynamic and kinematic free-surface boundary conditions, but the convolution approach provides an explicit expression for the necessary kinematic closure that couples the surface activity with the rest of the 3D flow. The convolution integrates over the still water surface and involves an impulse response function, which is computed prior to the time stepping. The impulse response function decays exponentially and in practice it has a horizontal footprint of just several water depths, which is typically only a tiny part of the entire computational domain. For an impulse in open water over mild-slope bathymetry, where the response footprint does not interfere with lateral boundaries or steep bottom slopes, the response may be computed analytically. For points in the vicinity of boundaries, structures or steep bathymetry, the determination of the impulse response function in general requires the solution of a local 3D Laplace problem. The horizontal size of this problem is limited by the footprint of the impulse response, and thus independent of the size of the entire computational domain. A physical-space version (Schäffer 2008a) of the perturbation scheme known from the high-order spectral method (West et. al. 1987, Dommermuth and Yue 1987) may provide wave nonlinearity to arbitrary order at the expense of additional convolutions. The present paper only considers linear waves, see Schäffer (2005) for preliminary nonlinear simulations in $1 \mathrm{DH}$.

\section{A SIMPLIFIED VERSION OF THE CONVOLUTION-TYPE WAVE MODEL}

The convolution approach relies on the time-stepping of the kinematic and dynamic free-surface boundary conditions with an explicit closure involving a convolution integral. Restricting this paper to linear waves, the kinematic and dynamic surface boundary conditions in $2 \mathrm{DH}$ read

$$
\begin{aligned}
\eta_{t} & =w_{0} \\
\phi_{0, t} & =-g \eta
\end{aligned}
$$

\footnotetext{
${ }^{1}$ SchäfferWaves, Sortedam Dossering 59 D st, DK-2100 Copenhagen $\varnothing$, Denmark
} 
where $w_{0}$ is the vertical particle velocity and $\phi_{0}$ is the velocity potential, both taken at the still water level, $\eta$ is the free surface elevation, $g$ is acceleration of gravity, and subscript $t$ denotes differentiation with respect to time.

The surface conditions can be integrated in time if $w_{0}$ is provided as a function of the other dependent variables. In a linear framework $w_{0}$ only depends on $\phi_{0}$ and the bathymetry, and it may be explicitly given in terms of a convolution integral involving an impulse response function, $R$. For locally constant depth, $h_{0}$, this writes

$$
w_{0}(x, y)=-\frac{1}{h_{0}} \int_{-\infty}^{\infty} \int_{-\infty}^{\infty} R(s) \nabla^{\prime 2} \phi_{0}\left(x^{\prime}, y^{\prime}\right) d y^{\prime} d x^{\prime}, \quad s=\frac{1}{h_{0}} \sqrt{\left(x-x^{\prime}\right)^{2}+\left(y-y^{\prime}\right)^{2}}
$$

While the integration limits are in theory infinite, they may in practice be taken as horizontal distances of just a few water depths, depending on the desired accuracy. This is due to the almost local support of the impulse response function, which decays exponentially in horizontal directions away from the impulse. If lateral boundaries exist within this distance then these will bound the integration limits further, while affecting the local variation of the impulse response function.

The impulse response function away form lateral boundaries and for constant depth

When lateral boundaries are more than several water depths away from the observation point and the water depth is constant, then the impulse response function is available in two different analytical forms. One is

$$
R(s)=\frac{1}{\pi} \sum_{n=0}^{\infty} \mathrm{K}_{0}\left((2 n+1) \frac{\pi}{2} s\right)
$$

where $\mathrm{K}_{0}$ is the modified Bessel function of the second kind of order zero. As $\mathrm{K}_{0}(r)$ $\sim \sqrt{\pi /(2 r)} \exp (-r)$, this shows the evanescent nature of $R$, which in the far field is dominated by the first term of the series. On the other hand all terms have a logarithmic singularity at $s=0$.

Another representation, which extracts the singular behavior in the first term, while the remaining series is regular, is given by

$$
R(s)=\frac{1}{2 \pi}\left(\frac{1}{s}+\sum_{n=1}^{\infty} \frac{(-1)^{n}}{\sqrt{\left(\frac{s}{2}\right)^{2}+n^{2}}}\right)
$$

Conversely, this version does not immediately display the exponential decay. While both of these representations are valuable components in the analytical framework of the theory, none of them are used in the discrete numerical model, see Schäffer $(2005,2009)$ and Schäffer $(2006,2010)$ for both constant-depth and mild-slope developments in 1DH and 2DH, respectively.

\section{The impulse response function near lateral boundaries for constant depth}

To determine the impulse response function near vertical lateral boundaries of complex shaped domains, Schäffer (2008b) devised an approach based on a series of Boundary Integral Equations (BIE), where the boundary was the perimeter (water line) of the computational domain. However, that approach was strictly limited to constant depth and therefore abandoned in favor an approach including the vertical extent of the lateral boundary as presented below, although still in a constant-depth context. Nevertheless, variable bathymetry is taken into account in the real-harbor example below. However, this is done using a temporary approach which is not entirely correct and hence not presented.

By Green's second identity the Laplace equation for the velocity potential $\phi$ leads to the Boundary Integral Equation (BIE)

$$
\alpha\left(\mathbf{x}_{3}\right) \phi\left(\mathbf{x}_{3}\right)=\int_{\Gamma}\left[\phi\left(\xi_{3}\right) G_{n_{\xi}}\left(\mathbf{x}_{3}, \xi_{3}\right)-G\left(\mathbf{x}_{3}, \xi_{3}\right) \phi_{n_{\xi}}\left(\xi_{3}\right)\right] d \Gamma, \quad \xi_{3} \in \Gamma
$$

Here $\alpha\left(\mathbf{x}_{3}\right)$ is zero for points external to the fluid. For the 2D case, $\alpha\left(\mathbf{x}_{3}\right)$ is $2 \pi$ for internal points and equals the interior angle, typically $\pi$, for observation points, $\mathbf{x}_{3}=(x, y, z)$, on the boundary $\Gamma$. In $3 \mathrm{D}, \alpha\left(\mathbf{x}_{3}\right)$ is $4 \pi$ for internal points and equal to the interior solid angle, typically $2 \pi$, for boundary points. Furthermore, $G$ is a Green's function for the Laplace equation, usually taken as the free-space Green's function and subscript $n$ indicates the derivative normal to the boundary. In the following, we chose a more elaborate Green's function that satisfies appropriate boundary conditions. For a domain 
that is constant in time, this has the advantage of transferring the complexity of the problem from solutions for the time-dependent quantities representing the velocity field to pre-computations of the time-independent Green's function and still-water-level impulse response function for an impulse in $\nabla^{2} \phi_{0}$.

Let the boundary $\Gamma$ be constituted by $\Gamma_{0}$ as contained in the still water level, $\Gamma_{a}$ as the lateral boundary, and $\Gamma_{b}$ as the bottom. For the Green's function, homogeneous Dirichlet boundary conditions are taken at $\xi_{3} \in \Gamma_{0}$, while homogeneous Neumann boundary conditions are taken at the solid boundaries, $\xi_{3} \in \Gamma_{a} \cup \Gamma_{b}$ i.e.

$$
\begin{array}{cccc}
G\left(\mathbf{x}_{3}, \xi_{3}\right)=0 & \text { for } & \xi_{3} \in \Gamma_{0} \\
G_{n_{\xi}}\left(\mathbf{x}_{3}, \xi_{3}\right)=0 & \text { for } & \xi_{3} \in \Gamma_{a} \\
G_{n_{\xi}}\left(\mathbf{x}_{3}, \xi_{3}\right)=0 & \text { for } & \xi_{3} \in \Gamma_{b}
\end{array}
$$

This yields

$$
\alpha\left(\mathbf{x}_{3}\right) \phi\left(\mathbf{x}_{3}\right)=\int_{\Gamma_{0}} \phi\left(\xi_{3}\right) G_{\zeta}\left(\mathbf{x}_{3}, \xi_{3}\right) d \Gamma+\int_{\Gamma_{a} \cup \Gamma_{b}} G\left(\mathbf{x}_{3}, \xi_{3}\right) \phi_{n_{\xi}}\left(\xi_{3}\right) d \Gamma
$$

Taking $\Gamma_{a}$ and $\Gamma_{b}$ be fixed solid boundaries by which homogeneous Neumann boundary conditions apply for the velocity potential, then the second integral vanishes and we have

$$
\alpha\left(\mathbf{x}_{3}\right) \phi\left(\mathbf{x}_{3}\right)=\int_{\Gamma_{0}} \phi\left(\xi_{3}\right) G_{\zeta}\left(\mathbf{x}_{3}, \xi_{3}\right) d \Gamma
$$

Let the water depth be constant and let $\Phi^{(a)}$ be a regular solution to the Laplace equation, then the Green's function may be expressed as

$$
G\left(\mathbf{x}_{3}, \boldsymbol{\xi}_{3}\right)=G^{(0)}\left(\mathbf{x}_{3}, \boldsymbol{\xi}_{3}\right)+\Phi^{(a)}\left(\mathbf{x}_{3}, \boldsymbol{\xi}_{3}\right)
$$

where $\Phi^{(a)}$ is due to the presence of the lateral boundaries. This leaves $G^{(0)}\left(\mathbf{x}_{3}, \boldsymbol{\xi}_{3}\right)$ as a Green's function appropriate for horizontally open areas of constant depth, $h_{0}$

$$
\begin{array}{ll}
G^{(0)}\left(\mathbf{x}_{3}, \xi_{3}\right)=0 & \text { for } \quad \zeta=0 \\
G_{\zeta}^{(0)}\left(\mathbf{x}_{3}, \xi_{3}\right)=0 & \text { for } \quad \zeta=-h_{0}
\end{array}
$$

The following boundary conditions for $\Phi^{(a)}$ ensure that the original conditions for the entire Green's function are satisfied

$$
\begin{array}{ll}
\Phi^{(a)}\left(\mathbf{x}_{3}, \boldsymbol{\xi}_{3}\right)=0 & \text { for } \zeta=0 \\
\Phi_{n_{\xi}^{(a)}}^{(a)}\left(\mathbf{x}_{3}, \xi_{3}\right)=-G_{n_{\xi}}^{(0)}\left(\mathbf{x}_{3}, \boldsymbol{\xi}_{3}\right) & \text { for } \xi_{3} \in \Gamma_{a} \\
\Phi_{\zeta}^{(a)}\left(\mathbf{x}_{3}, \boldsymbol{\xi}_{3}\right)=0 & \text { for } \zeta=-h_{0}
\end{array}
$$

Inspired by previous, unpublished comparison between the convolution approach and the boundary integral equation method, we now introduce a function $Q(r, z)$ given by an infinite series of vertically aligned, alternating sources and sinks with a distance of $2 h_{0}$ between consecutive singularities,

$$
Q(r, z)=\sum_{n=-\infty}^{\infty}(-1)^{n} \frac{1}{\sqrt{r^{2}+\left(z+2 n h_{0}\right)^{2}}}=\frac{2}{h_{0}} \sum_{m=0}^{\infty} \mathrm{K}_{0}\left(\kappa_{m} r\right) \cos \left(\kappa_{m} z\right), \quad \kappa_{n} \equiv \frac{\pi}{2 h_{0}}(1+2 m),
$$

where the last expression is a Fourier series and $\mathrm{K}_{0}$ as before is the modified Bessel function of second kind and order zero. For later use, we note that

$$
\begin{aligned}
Q_{\zeta}(r, \zeta)=0 & \text { for } \quad \zeta=0 \\
Q(r, \zeta)=0 & \text { for } \zeta=-h_{0}
\end{aligned}
$$

With $r=|\mathbf{x}-\xi|$, where $\mathbf{x}$ is an observation point and $\xi$ is an integration point both at the still water level, $Q(r, z-\zeta)$ is in itself a Green's function, but it does not satisfy the boundary conditions (11). In fact comparison between (11) and (14) shows that Neumann and Dirichlet conditions have swapped between the surface and the bottom. However the two conditions in (11) can be satisfied by adding the image in the bottom, $\zeta=-h_{0}$, or equivalently, by subtracting the image in $\zeta=0$. Thus, we take

by which

$$
G^{(0)}\left(\mathbf{x}_{3}, \xi_{3}\right)=Q(r, z+\zeta)-Q(r, z-\zeta), \quad r=|\mathbf{x}-\xi|
$$




$$
G^{(0)}\left(\mathbf{x}_{3}, \boldsymbol{\xi}_{3}\right)=-\frac{4}{h_{0}} \sum_{n=0}^{\infty} \mathrm{K}_{0}\left(\kappa_{n} r\right) \sin \left(\kappa_{n} z\right) \sin \left(\kappa_{n} \zeta\right)
$$

In general, this is a Green's function with an appropriate singularity at $\zeta=z$. However, an exception occurs when the observation point is located at $z=0$, for which sources and sinks coalesce and $G^{(0)}$ vanishes for all values of $\xi_{3}$. This is of fundamental concern, since it is exactly the point $z=0$ that is needed for the closure of the time stepping problem given by the dynamic and the kinematic free surface boundary conditions. However, the integral representation (9) remains meaningful as

$$
\left.G_{\zeta}^{(0)}\left(\mathbf{x}_{3}, \xi_{3}\right)\right|_{\zeta=0} \rightarrow \pm 4 \pi \delta(\mathbf{x}-\xi) \quad \text { for } \quad z \rightarrow 0^{\mp}
$$

while the observation point stays internal by which $\alpha\left(\mathbf{x}_{3}\right)=4 \pi$. In this limit, the two terms on the right-hand-side of (15) contribute to (17) by equal amounts and each contribution comes from $n=0$ in (13) while positive and negative index terms pair up to zero. For $n=0$ the two equal contributions add up to $-2 z /\left(r^{2}+z^{2}\right)^{3 / 2}$, which provides the limit in (17).

Taking the $z$-derivative of (9) yields

$$
\begin{aligned}
\alpha\left(\mathbf{x}_{3}\right) w\left(\mathbf{x}_{3}\right) & =\int_{\Gamma_{0}} \phi\left(\xi_{3}\right) G_{\zeta z}\left(\mathbf{x}_{3}, \xi_{3}\right) d \Gamma \\
& =\int_{\Gamma_{0}} \phi\left(\xi_{3}\right) G_{\zeta z}^{(0)}\left(\mathbf{x}_{3}, \xi_{3}\right) d \Gamma+\int_{\Gamma_{0}} \phi\left(\xi_{3}\right) \Phi_{\zeta z}^{(a)}\left(\mathbf{x}_{3}, \xi_{3}\right) d \Gamma
\end{aligned}
$$

where the second integral is regular, while $G_{\zeta z}^{(0)}$ makes the first integral hypersingular in case of $z=0$. To eliminate hypersingularity, we proceed as follows. From (16), we get

$$
G_{\zeta z}^{(0)}\left(\mathbf{x}_{3}, \xi_{3}\right)=-\frac{4}{h_{0}} \sum_{m=0}^{\infty} \kappa_{m}^{2} \mathrm{~K}_{0}\left(\kappa_{m} r\right) \cos \left(\kappa_{m} z\right) \cos \left(\kappa_{m} \zeta\right)
$$

and using the identity ( $\mathrm{K}_{0}$ satisfies the modified Helmholtz equation in $2 \mathrm{DH}$ )

$$
\kappa_{m}^{2} \mathrm{~K}_{0}\left(\kappa_{m} r\right)=\nabla_{\xi}^{2} \mathrm{~K}_{0}\left(\kappa_{m} r\right), \quad \nabla_{\xi}=\left(\frac{\partial}{\partial \xi}, \frac{\partial}{\partial \psi}\right), \quad r>0,
$$

the kernel becomes

$$
G_{\zeta z}^{(0)}\left(\mathbf{x}_{3}, \xi_{3}\right)=-\frac{4}{h_{0}} \nabla_{\xi}^{2} \sum_{m=0}^{\infty} \mathrm{K}_{0}\left(\kappa_{m} r\right) \cos \left(\kappa_{m} z\right) \cos \left(\kappa_{m} \zeta\right)
$$

Comparing (21) with (13), we may write $G_{\zeta z}^{(0)}$ in terms of $Q$ as

$$
G_{\zeta z}^{(0)}\left(\mathbf{x}_{3}, \boldsymbol{\xi}_{3}\right)=-\nabla_{\xi}^{2}[Q(r, z+\zeta)+Q(r, z-\zeta)]
$$

by which

$$
\left.G_{\zeta z}^{(0)}\left(\mathbf{x}_{3}, \xi_{3}\right)\right|_{\zeta=0}=-2 \nabla_{\xi}^{2} Q(r, z)
$$

For vertical lateral boundaries and constant depth, $\Phi^{(a)}$ must have the same vertical structure as $G^{(0)}$ and along the lines of (15) we introduce a function $\Theta$ with the property that

$$
\Phi^{(a)}\left(\mathbf{x}_{3}, \boldsymbol{\xi}_{3}\right)=\Theta(r, z+\zeta)-\Theta(r, z-\zeta), \quad r=|\mathbf{x}-\boldsymbol{\xi}|
$$

This leads to a counterpart to (23),

$$
\left.\Phi_{\zeta z}^{(a)}\left(\mathbf{x}_{3}, \xi_{3}\right)\right|_{\zeta=0}=-2 \nabla_{\xi}^{2} \Theta(r, z)
$$

and substituting both in (18) yields

$$
\alpha\left(\mathbf{x}_{3}\right) w\left(\mathbf{x}_{3}\right)=-2 \int_{\Gamma_{0}} \phi\left(\xi_{3}\right) \nabla_{\xi}^{2}[Q(r, z)+\Theta(r, z)] d \Gamma
$$

While the basic boundary integral equation, (6), is based on Green's $2^{\text {nd }}$ identity in three dimensions, we now proceed by applying the same theorem, but in two dimensions, to eliminate the hypersingular behavior for $z=0$. This yields

$$
\alpha\left(\mathbf{x}_{3}\right) w\left(\mathbf{x}_{3}\right)=-2 \int_{\Gamma_{0}} \nabla_{\xi}^{2} \phi\left(\xi_{3}\right)[Q(r, z)+\Theta(r, z)] d \Gamma
$$

where two 'waterline' integrals originating from the application of Green's $2^{\text {nd }}$ identity have vanished due to lateral homogeneous Neumann boundary conditions for both $\phi$ and $Q+\Theta$. For observation points at the still water level, $z=0,(27)$ becomes 


$$
\alpha(\mathbf{x}) w_{0}(\mathbf{x})=-2 \int_{\Gamma_{0}} \nabla_{\xi}^{2} \phi\left(\xi_{3}\right)[Q(r, 0)+\Theta(r, 0)] d \Gamma
$$

which provides the desired explicit closure for time-stepping the free-surface boundary conditions. Here $Q$ is given by (13), while $\Theta$ is the solution to a local problem governed by the homogeneous 3D Laplace equation and satisfying the boundary conditions

$$
\begin{array}{ll}
\Theta_{\zeta}(r, \zeta)=0 & \text { for } \quad \zeta=0 \\
\Theta_{n_{\xi}}(r, \zeta)=-Q_{n_{\xi}}(r, \zeta) & \text { for } \quad \xi_{3} \in \Gamma_{a} \\
\Theta(r, \zeta)=0 & \text { for } \quad \zeta=-h_{0}
\end{array}
$$

For observation points located away from lateral boundaries $\Theta$ vanishes and with $\alpha(\mathbf{x})=4 \pi$ this makes (28) reduce to the original convolution expression (3) by virtue of the relation $2 \pi R(s)=h_{0} Q(r, 0)$ as found by comparing (4) with (13).

\section{EXAMPLES}

\section{Diffraction around a semi-infinite vertical barrier}

As a canonical example, the left panel of Figure 1 shows the classical Sommerfeld diffraction case (Sommerfeld, 1896, Penney and Price, 1952) and the right panel illustrates the problem to be solved when the incident wave is replaced by the undisturbed impulse response function. Figure 2 exemplifies the numerical and analytical solution in terms of what needs to be added to the 'incident' impulse response function to account for the existence of the barrier i.e. $\Theta(r, 0)$. The result is an odd function of the distance from the barrier and it only shows appreciable size in a region close to both the breakwater and the impulse.

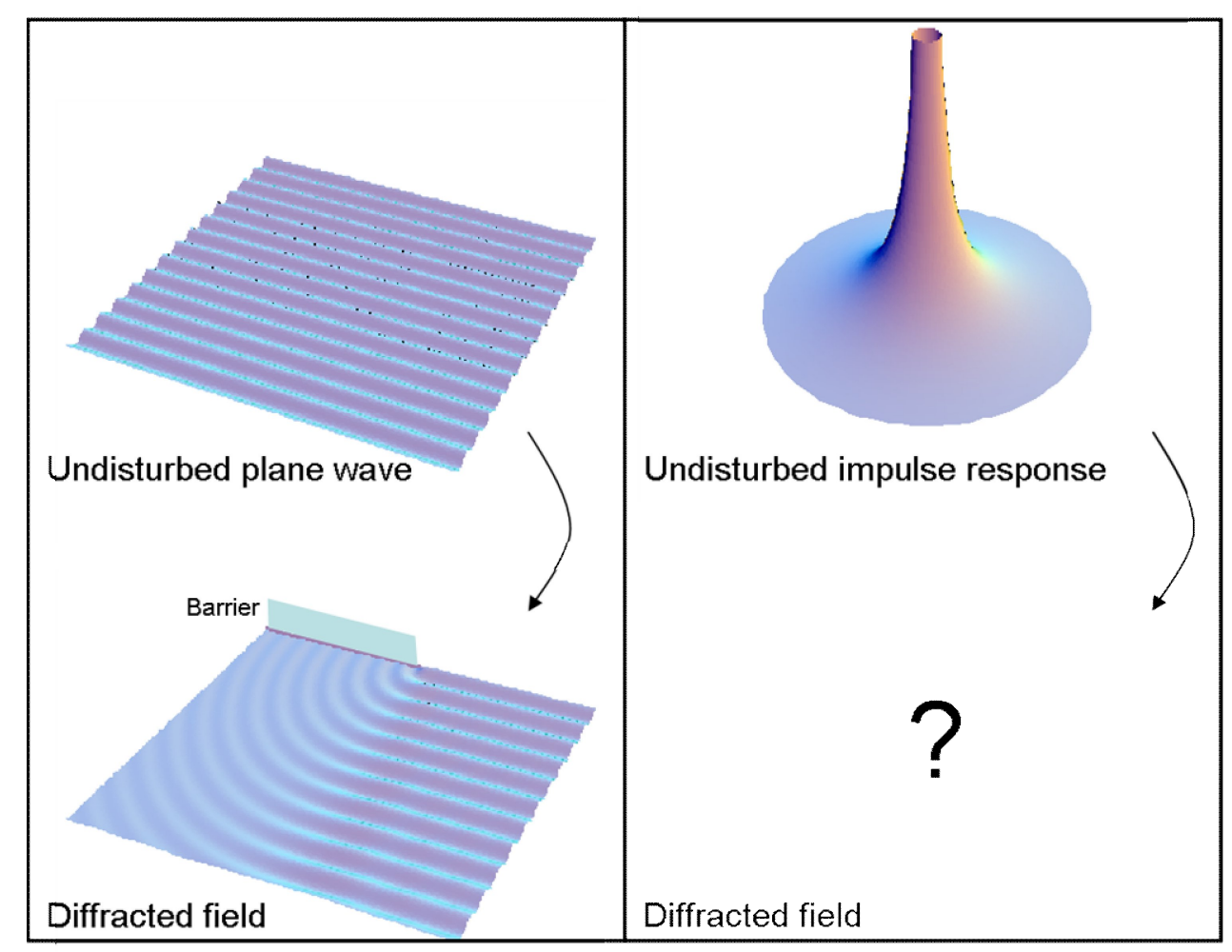

Figure 1 - Example of diffraction problem for a semi-infinite vertical barrier. Left panel shows the classical Sommerfeld diffraction case. Right panel illustrates the quest for an analogous result. 


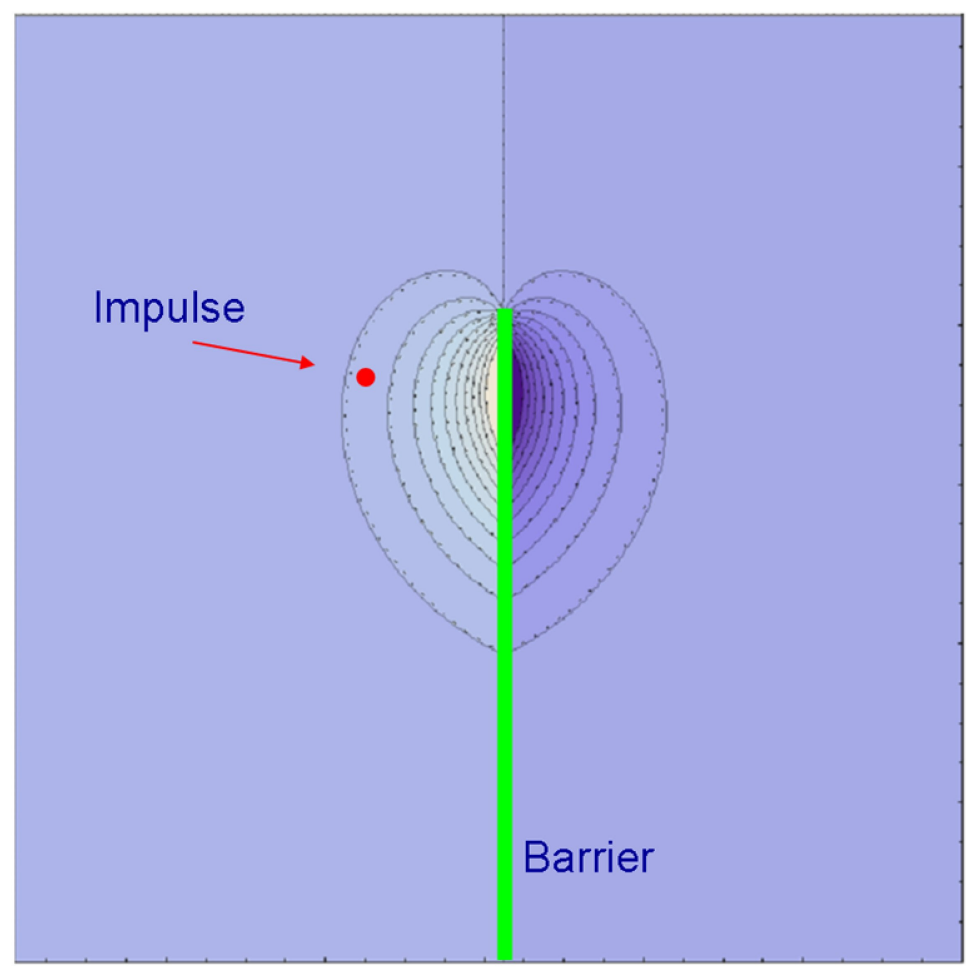

Figure 2 - Contours in the horizontal plane of the additional impulse response, $\Theta(r, 0)$, caused by the barrier. Red dot: Impulse location. Green line: vertical barrier. Full lines: analytical solution. Dashed lines: Finite-difference Laplace solution.

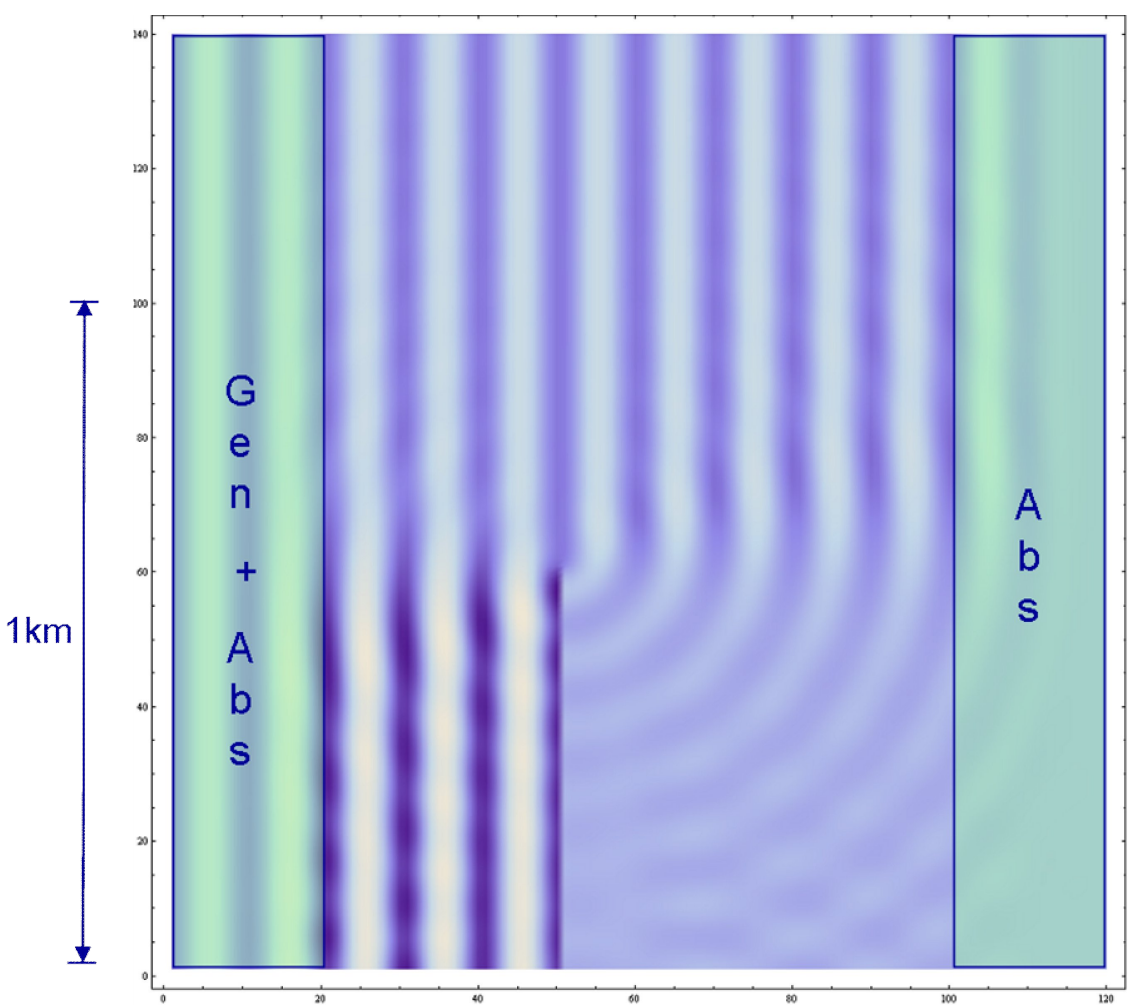

Figure 3 - Surface elevation snapshot for the Sommerfeld diffraction case. Waves are incident from the left, reflected from and diffracted around the barrier in the lower middle of the plot and absorbed to the right. Wave period $8 \mathrm{~s}$, wave length about $100 \mathrm{~m}$, water depth $40 \mathrm{~m}$, grid size $10 \mathrm{~m}$ by $10 \mathrm{~m}$, domain size 120 by 140 points. 
Recall that $r=|\mathbf{x}-\boldsymbol{\xi}|$, where $\mathbf{x}$ is an observation point and $\xi$ is an integration point both at the still water level. For constant depth, $Q(r, 0)$ is similar everywhere, but this is not the case for $\Theta(r, 0)$, which has to be evaluated for observation points located anywhere near the barrier. The underlying computation of $\Theta(r, z)$ is made through a finite-difference solution to the 3D Laplace problem in the barrier vicinity. For this specific example, the entire barrier neighborhood was easily treated as a single domain resulting in a linear system with one sparse matrix and as many right-hand-sides as there are observation points. As a one-time computation taking place prior to the time-stepping of the wave propagation problem, the associated computational effort was insignificant. With the impulse response function in place, including the account for the barrier, the wave evolution in time proceeds using an explicit fourth-order Runge Kutta integration in time (RK4), but also a fully explicit procedure in space. For monochromatic incident waves, Figure 3 shows a snapshot of the surface elevation after the wave field has developed into a time-periodic field. The resemblance to the Sommerfeld diffraction solution is evident and a quantitative comparison (not shown) confirms the good agreement. Nonetheless, the computation is preliminary and further improvements are anticipated.

\section{Wave penetration in a real harbor}

Taking the Danish Hanstholm Harbor as example, Figure 4 shows the footprint of the impulse response function for various water depths. The full circles with depth labels illustrate footprints unaffected by the lateral boundaries. The semicircle and circle at the breakwater show the respective footprints associated with observation points right next to the boundary and at the breakwater head.

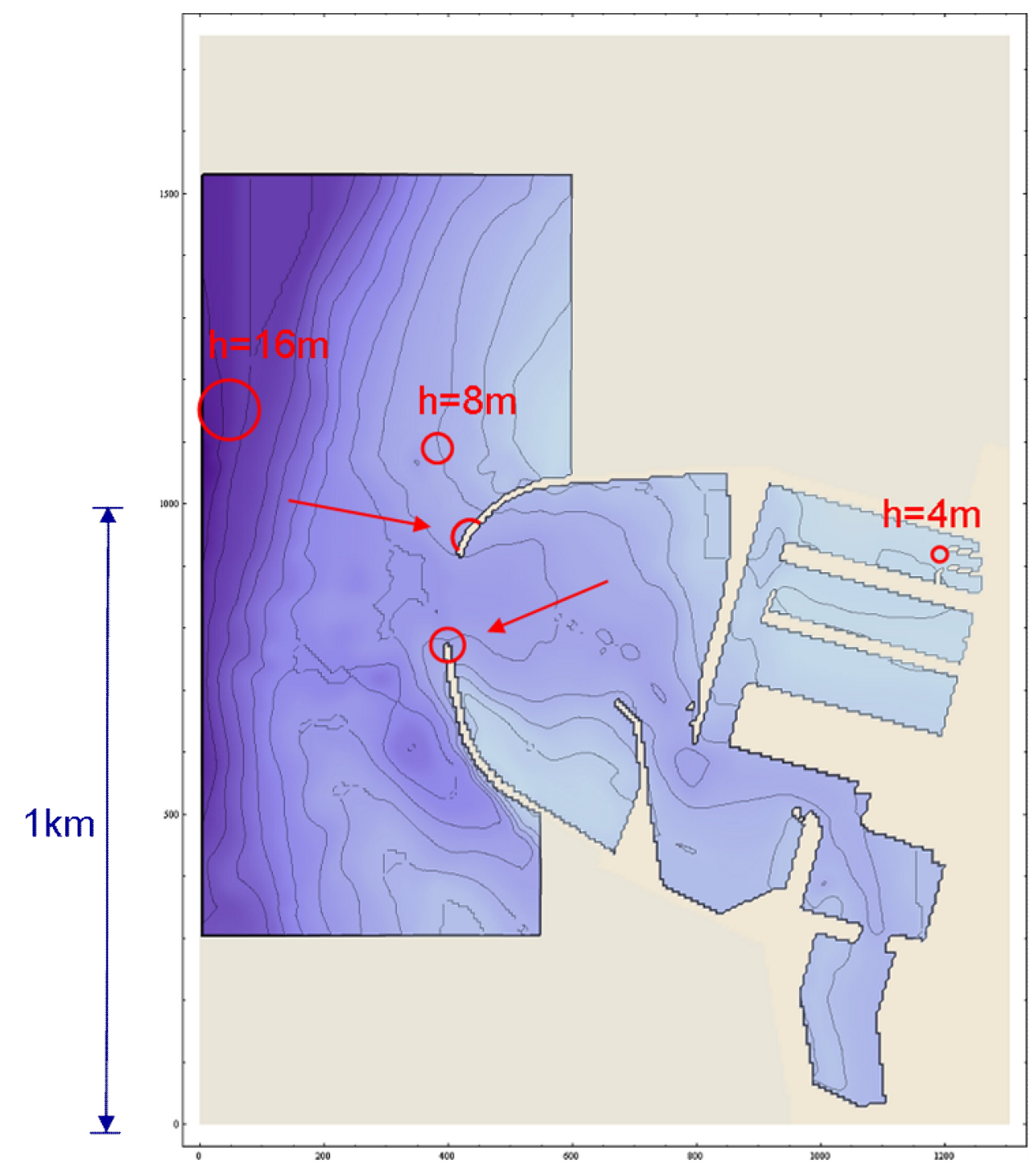

Figure 4 - Bathymetry and layout of Hanstholm Harbor with illustrations of the footprint of the impulseresponse function at various water depths. 
Figure 5 shows a result of a very preliminary simulation with waves incident from the left. Although the picture may look like a snapshot of surface elevation it actually shows the maximum elevation over a couple of wave periods. The reason for the large variation is that no means of absorption has yet been applied at the lateral boundaries. This means that all structures are fully reflective. While this is definitely not a physically relevant condition, it does provide a more demanding test of the numerical model stability. No stability issues were encountered.

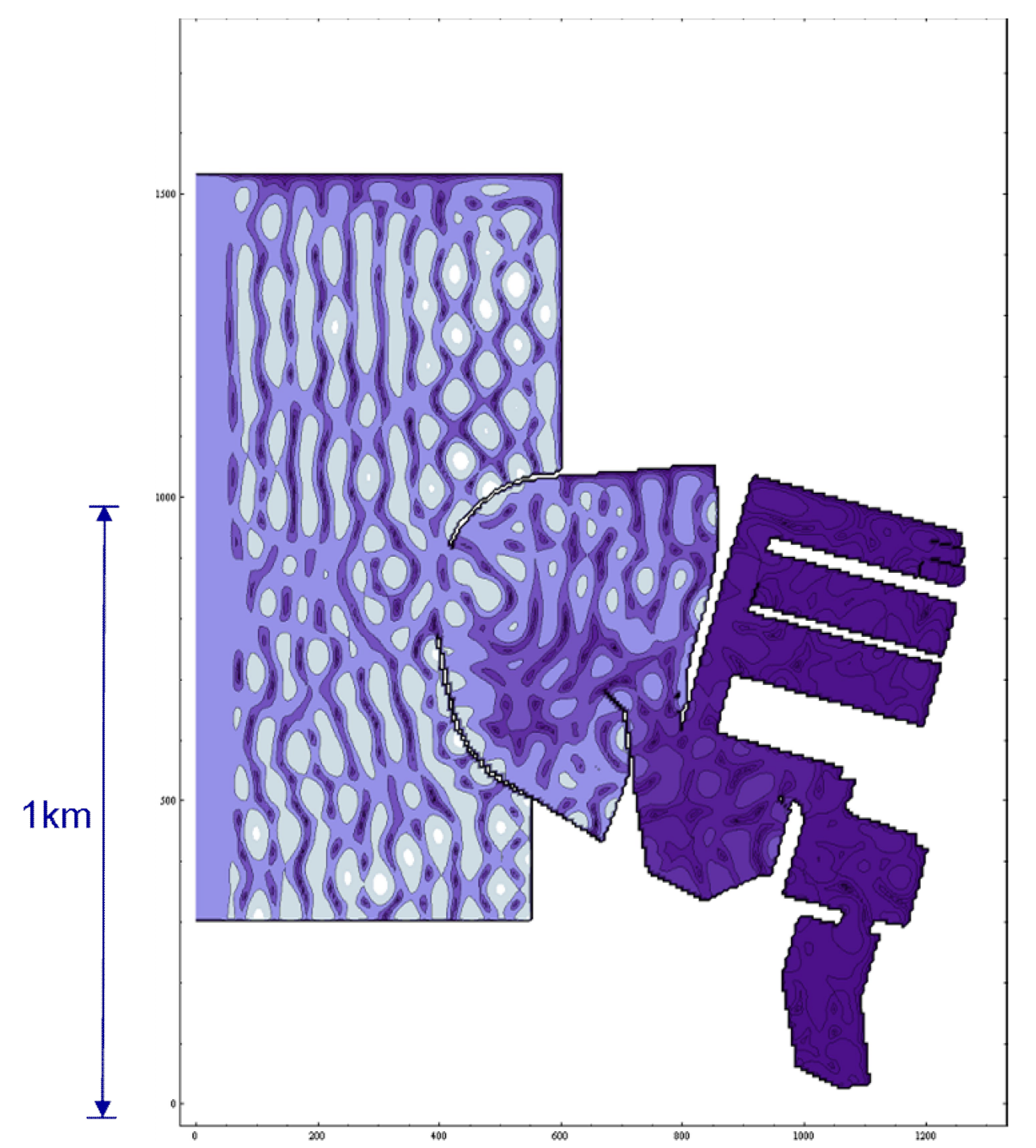

Figure 5 - Contours of maximum surface elevation of a very preliminary regular-wave simulation with full reflection at all boundaries except for the wave generation and absorption zone to the left.

\section{CONCLUDING REMARKS}

This paper only shows modeling examples that can be successfully treated by existing Boussinesqtype models. However, for the convolution-type approach these examples serve their purpose as test cases (still to be completed) and provide necessary steps that must be taken on the way towards a model with a wider scope than most if not all existing deterministic wave models. The idea of a 3D model at $2 \mathrm{D}$ effort as launched decades ago for boundary element models may finally turn out to be satisfied by the related convolution-type approach. 


\section{ACKNOWLEDGMENTS}

Support from the Danish Council for Independent Research | Technology and Production Sciences (FTP 09-065781) is greatly appreciated. DHI is acknowledged for making port layout and bathymetric data available.

\section{REFERENCES}

Dommermuth, D.G. and Yue, D.K.Y. (1987). A high-order spectral method for the study of nonlinear gravity waves. J. Fluid Mech. 184, 267-288.

Penney, W.G. and Price, A.T. (1952). Part I. The diffraction theory of sea waves and the shelter afforded by breakwaters. Phil. Trans. Roy. Soc. Lond. A, Mathematical and Physical Sciences, 244 (882), 236-253.

Schäffer, H.A. (2005). A convolution method for 1-D nonlinear dispersive wave transformation over a mild-slope bottom. Waves '05: Proceedings of $5^{\text {th }}$ Int. Symp. On Ocean Wave Measurement and Analysis, ASCE.

Schäffer, H.A. (2006). Progress on a convolution method for nonlinear dispersive wave transformation over gently varying bathymetry. Proceedings of $30^{\text {th }}$ International Conference on Coastal Engineering, ASCE.

Schäffer, H.A. (2008a). Comparison of Dirichlet-Neumann operator expansions for nonlinear surface gravity waves. Coastal Engineering, 55, 288-294.

Schäffer, H.A. (2008b). Toward a fast convolution-type wave model in non-rectangular domains. Proceedings of $31^{\text {st }}$ International Conference on Coastal Engineering, ASCE.

Schäffer, H.A. (2009). A fast convolution approach to the transformation of surface gravity waves: Linear waves in 1DH. Coastal Engineering, 56, 517-533.

Schäffer, H.A. (2010). Global error control and cpu-time minimization in deterministic wave models exemplified by a fast convolution-type model. Proceedings of $32^{\text {nd }}$ International Conference on Coastal Engineering, ASCE.

Sommerfeld, A. (1896). Mathematische theorie der diffraction. Math. Ann. 47, 317-374.

West, B.J., Brueckner, K.A., Janda, R.S., Milder D.M. and Milton, R.L (1987). A new numerical method for surface hydrodynamics. J. Geophys. Res. 92(C11), 11803-11824. 